(W)

Check for

updates

Cite as

Nano-Micro Lett.

(2021) 13:54

Received: 27 August 2020

Accepted: 2 November 2020

Published online: 8 January 2021

(C) The Author(s) 2021

\section{Chemical Coupled PEDOT:PSS/Si Electrode: Suppressed Electrolyte Consumption Enables Long-Term Stability}

Xuejiao Liu ${ }^{1}$, Zhixin $\mathrm{Xu}^{1}$, Asma Iqbal ${ }^{1}$, Ming $\mathrm{Chen}^{1}{ }^{1}$, Nazakat Ali ${ }^{1}$, CheeTongJohn Low ${ }^{2}$, Rongrong Qi ${ }^{1 凶}$, Jiantao Zai ${ }^{1 凶}$, Xuefeng Qian ${ }^{1 凶}$

\title{
HIGHLIGHTS
}

- $\gamma$-Glycidoxypropyl trimethoxysilane (GOPS) was incorporated in Si/PEDOT:PSS electrodes to construct a flexible and conductive artificial solid-electrolyte interphase (SEI).

- XPS $\mathrm{Ar}^{+}$etching depth analysis proved that the addition of GOPS is conducive to forming a more stable SEI.

- A full battery assembled with NCM 523 cathode delivers a high energy density of $520 \mathrm{Wh} \mathrm{kg}^{-1}$, offering good stability.

\begin{abstract}
Huge volume changes of Si during lithiation/delithiation lead to regeneration of solid-electrolyte interphase (SEI) and consume electrolyte. In this article, $\gamma$-glycidoxypropyl trimethoxysilane (GOPS) was incorporated in Si/PEDOT:PSS electrodes to construct a flexible and conductive artificial SEI, effectively suppressing the consumption of electrolyte. The optimized electrode can maintain $1000 \mathrm{mAh} \mathrm{g}^{-1}$ for nearly 800 cycles under limited electrolyte compared with 40 cycles of the electrodes without GOPS. Also, the optimized electrode exhibits excellent rate capability. The use of GOPS greatly improves the interface compatibility between Si and PEDOT:PSS. XPS $\mathrm{Ar}^{+}$etching depth analysis proved that the addition of GOPS is conducive to forming a more stable SEI. A full battery assembled with NCM 523 cathode delivers a high energy density of $520 \mathrm{Wh} \mathrm{kg}^{-1}$, offering good stability.
\end{abstract}

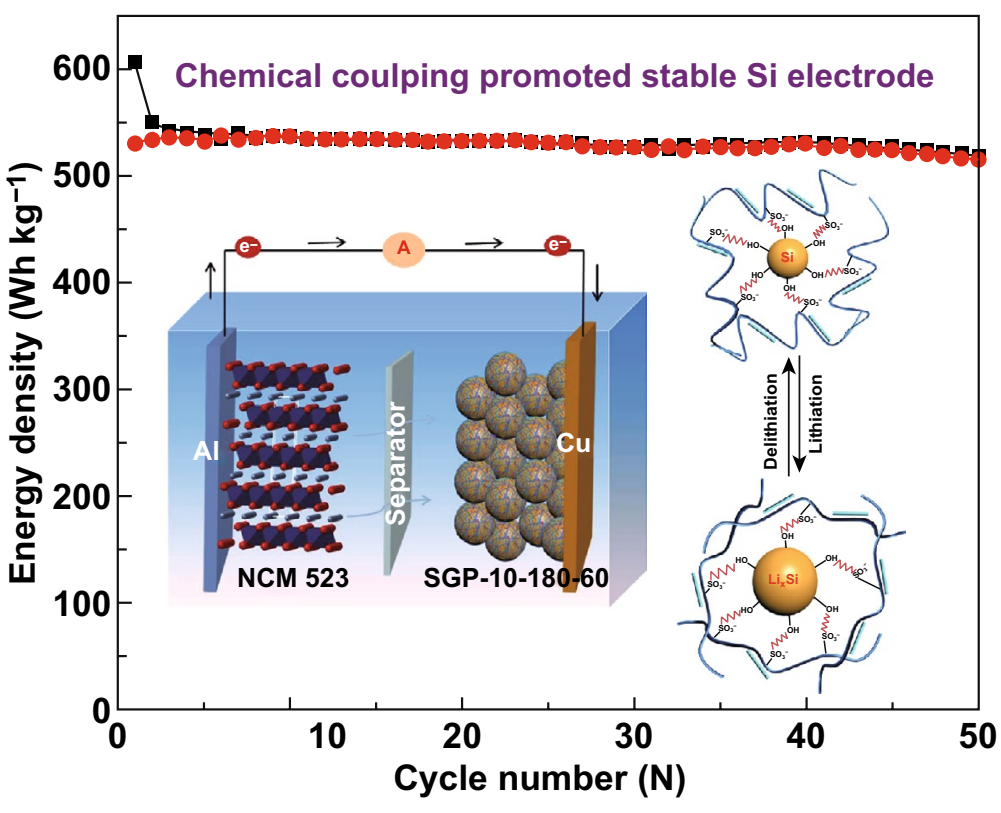

KEYWORDS Conductive binder; PEDOT:PSS; Cross-link; XPS depth analysis; Stable SEI; Lithium-ion batteries

Rongrong Qi, rrqi@sjtu.edu.cn; Jiantao Zai, zaijiantao@sjtu.edu.cn; Xuefeng Qian, xfqian@ @jtu.edu.cn

1 School of Chemistry and Chemical Engineering and State Key Laboratory of Metal Matrix Composites, Shanghai Electrochemical Energy Devices

Research Center, Shanghai Jiao Tong University, Shanghai 200240, P. R. China

2 Warwick Electrochemical Engineering Group, Energy Innovation Centre, WMG, University of Warwick, Coventry CV4 7AL, UK 


\section{Introduction}

$\mathrm{Si}$, as one of the most promising anode materials for lithium-ion batteries, has been investigated for decades due to its highest theoretical specific capacity $\left(4200 \mathrm{mAh} \mathrm{g}^{-1}\right)[1,2]$. However, Si suffers from huge volume changes $(>300 \%)$ during lithiation/delithiation, which causes active material pulverization and exfoliation from the collector. Both problems can now be relieved by nano-Si [3-5] and surface-bonded binders [6]. However, there will be a solid electrolyte interface (SEI) on the anode surface as the strong reducibility leads to the reduction and decomposition of the electrolyte. The formation of SEI helps to avoid direct contact between the active material and the electrolyte. But huge volume expansion of Si during lithiation also causes SEI rupture, so additional electrolyte and $\mathrm{Li}^{+}$are irreversibly consumed repairing regenerations, which severely limits the Coulombic efficiency and cyclic stability. This problem is usually ignored in most anode studies due to excessive electrolyte in half-cell designs with Li metal as a counter electrode. To achieve high energy density in the actual full battery, the amount of electrolyte will be limited. However, SEI regeneration will overconsume electrolytes, resulting in sudden battery death. An ideal solution would be creating an artificial SEI on Si via an organic/ polymer coating layer [7-10], which can effectively control the contact between electrolyte and active material. Nevertheless, the artificial SEI is usually insulating that cannot transfer both $\mathrm{Li}^{+}$and electrons well. Thus, it is necessary to construct a flexible artificial SEI that is dual conductive for electronics and ions.

Conventional Si electrodes contain nearly $40 \mathrm{wt} \%$ inactive materials, including conductive additive and insulating binders, such as PAA [11], CMC, or alginate [12]. Conducting polymers with dual functions as a binder and conducting additives have been developed for Si anodes [13-16]. Among that, PEDOT is an excellent electronic conductor due to its rigid conjugated backbone $[17,18]$ and has been explored in lithium-ion batteries via in situ polymerization of EDOT [19-21]. However, poor ions diffusion ability and bad elasticity of pure conjugate rigid backbone may lead to poor cycling stability. Commercial PEDOT:PSS is a mixed ion-electron conductor. Besides, self-healing properties and improved elasticity of PEDOT:PSS make it more suitable as a binder for $\mathrm{Si}$ anodes $[22,23]$. Previous reports focused on improving mechanical performance or electrical conductivity of PEDOT:PSS itself. Huo's group did cross-link of D-sorbitol and vinyl acetateacrylic onto PEDOT:PSS chains to form a highly stretchable conductive glue and used as a high-performance binder [23]. Zhou's group assembled PEO and PEI onto PEDOT:PSS chains and used as a binder with high ion and electron conductivities [24]. Our previous work used multivalent ion-crosslinking PEDOT:PSS to enhance the strength by forming 3D structured polymers [25].

Although the above researches have alleviated the disadvantages of the Si negative electrode to a certain extent, these are mainly focused on optimizing the mechanical performance of binder but ignored the interface interaction with Si. The hydrophobicity and negativity of Si results in incompatible interfaces and charge repulsion, limiting the development of PEDOT:PSSbased binders. Therefore, improvements in interfacial properties may play a crucial role [26-28]. In another work, we improved interfacial compatibility and conductivity by glycerol crosslinking [12], but the interaction between $\mathrm{Si}$ and glycerol was relatively weak and needed to be further strengthened.

In this paper, considering the overall electrode structure, a silane coupling agent, $\gamma$-glycidoxypropyl trimethoxysilane (GOPS), was incorporated as a bridge between the Si and PEDOT:PSS to form a spatial network structure for the highperformance electrode (SGP electrode). The use of GOPS greatly improves the interface compatibility between active material and binder. The SGP-10-180-60 electrode exhibited higher peeling force, smaller $R_{\mathrm{SEI}}$ resistance after cycling. XPS $\mathrm{Ar}^{+}$etching depth analysis proved that the addition of GOPS is conducive to forming a more stable SEI. As a result, the obtained SGP-10-180-60 electrode showed better cycling stability and rate capability. After cross-linking, the SGP-10180-60 electrode could cycle validly for $~ 800$ times (compared with 40 cycles for the electrodes before cross-linking) under a set specific capacity of $1000 \mathrm{mAh} \mathrm{g}^{-1}$. Full battery composed of SGP-10-180-60 anode with NCM 523 cathode exhibited a high capacity of $\sim 1.5 \mathrm{mAh} \mathrm{cm}^{-2}$ and displayed ICE of $87.6 \%$. The initial resultant specific energy is $530 \mathrm{Wh} \mathrm{kg}^{-1}$ (based on the total weight of anode and cathode), and after 60 cycles, it maintained $485 \mathrm{Wh} \mathrm{kg}^{-1}$.

\section{Experimental Section}

\subsection{Materials}

PEDOT:PSS (1.4 wt\% in water) was obtained from Adamas, and the Si NPs were supplied by Jingxing alloy welding materials co. LTD. ( $\gamma$-Glycidyloxypropyl) trimethoxysilane 
(GOPS) was obtained from Adamas-beta. Polyacrylic acid (PAA) was supplied by Alfa-Aesar. All the chemicals were used directly without further purification.

\subsection{Preparation of a Series of SGP Electrodes}

In a typical experiment, a mixture of $80 \mathrm{mg}$ of Si NPs, $20 \mathrm{mg}$ (1.428 mL) of PEDOT:PSS, and $10 \mathrm{mg}$ of GOPS was stirred to form an uniform slurry. After stirring for $6 \mathrm{~h}$, the slurry was coated on a copper foil using a doctor blade method and dried overnight at $80{ }^{\circ} \mathrm{C}$. Then, the as-prepared electrodes were further heat-treated at $180{ }^{\circ} \mathrm{C}$ for $60 \mathrm{~min}$. The resulting electrode is denoted as SGP-10-180-60 electrode. For comparison, the electrodes with $5 \mathrm{mg}$ GOPS and $20 \mathrm{mg}$ GOPS were also prepared using the same method except for the mass of GOPS, and were denoted as SGP-5-180-60 and SGP-20180-60, respectively. Similarly, the electrodes of SGP-10160-60, SGP-10-200-60, SGP-10-180-0, SGP-10-180-10, and SGP-10-180-120 were also designed following same methodology. The Si-PEDOT:PSS electrode was prepared by the same method but without addition of GOPS cross-linker.

\subsection{Preparation of Si-PAA-SP Electrode}

In a typical experiment, a mixture of $80 \mathrm{mg}$ Si nanoparticles, $20 \mathrm{mg}$ PAA binder, and $10 \mathrm{mg}$ super $\mathrm{P}$ was stirred to form a uniform slurry. After stirring for $6 \mathrm{~h}$, the slurry was coated on a copper foil using a doctor blade method and dried overnight at $80^{\circ} \mathrm{C}$.

\subsection{Preparation of SGP-10-180-60||NCM 523 Full Batteries}

NCM 523 electrodes with a mass loading of $10 \mathrm{mg} \mathrm{cm}^{-2}$ were supplied by Ningbo Shanshan Co., Ltd. The SGP-10180-60 anode and NCM 523 cathode had been activated by pre-cycling 3 times at low current (anode: $200 \mathrm{~mA} \mathrm{~g}^{-1}$, cathode: $20 \mathrm{~mA} \mathrm{~g}^{-1}$ ) before assembling into full battery.

\subsection{Characterizations}

$\mathrm{X}$-ray diffraction measurements were carried out on Shimadzu XRD-6000 using $\mathrm{Cu} \mathrm{K} \alpha$ radiation $(\lambda=1.5418 \AA ̊)$, and $2 \theta$ from 10 to $80^{\circ}$ with a scan rate of $6^{\circ} \mathrm{min}^{-1}$. TEM (PHILIPS, Tecnai 12) and FESEM (JEOL, JSM-6700F or FESEM, Hitachi, S-4800) were conducted to study the morphological features of the samples. Raman spectroscopy was conducted on a Jobin-Yvon LabRam HR80 spectrometer to examine the chemical composition using a $532 \mathrm{~nm}$ laser. Fourier transform infrared spectrometer (FTIR) spectra were obtained by Nicolet 6700 spectrometer. X-ray photoelectron spectroscopy (XPS) was performed on a VG Scientific ESCLAB 220 iXL X-ray photoelectron spectrometer. Peeling test was measured with a universal electromechanical tester (Instron 4465) to evaluate the binder strength, and an electrode sample prepared in $30 \mathrm{~mm}$ width and $80 \mathrm{~mm}$ length was attached to $3 \mathrm{M}$ tape.

\subsection{Electrochemical Measurements}

Electrochemical tests were conducted using CR2016 coin half cells. Electrochemical cells were assembled with asprepared electrodes, metallic lithium foil as counter electrode. The electrolyte is $1 \mathrm{M}$ solution of $\mathrm{LiPF}_{6}$ in a mixture of ethylene carbonate (EC)/diethylene carbonate (DEC) (1:1, vol\%) with $10 \%$ fluoroethylene carbonate (FEC) was used as the additive and the separator is obtained from Celgard 2400. The weight of each piece of electrode is around $1.0 \mathrm{mg}$. The SGP-10-180-60 || NCM 523 full batteries were assembled by similar method. The half-cells were galvanostatic discharged/charged in the fixed voltage range of $0-1.5 \mathrm{~V}$, and full cells were tested in $2.8-4.25 \mathrm{~V}$ by the battery testing system (LAND CT2001A model, Wuhan jinnuo Electronics, China). Cyclic voltammetry (CV) was implemented at $0.2 \mathrm{mV} \mathrm{s}^{-1}$ between 0.01-2.0 V. Electrochemical impedance spectroscopy (EIS) was performed on a CHI 650E electrochemical workstation in the frequency range from $100 \mathrm{kHz}$ to $0.1 \mathrm{~Hz}$.

\section{Results and Discussion}

\subsection{Electrochemical Performance of Different Electrodes}

In this work, commercial Si nanoparticles were used as active material without further purification (Fig. S1). To investigate the effects of different cross-linking conditions 
on cyclic stability, the galvanostatic cycling tests of SGP anodes at a diverse cross-linking time (Fig. S2a), different cross-linking temperatures (Fig. S2b), and different GOPS amounts (Fig. S2c) were conducted at $1.0 \mathrm{~A} \mathrm{~g}^{-1}$. It is evident from Fig. S2 that the best cyclic stability is obtained when the cross-linking process was kept $60 \mathrm{~min}$ at $180{ }^{\circ} \mathrm{C}$, and $10 \mathrm{mg}$ GOPS added. After 200 cycles, the SGP-10-180-60 electrode can maintain a high reversible specific capacity of $1957.6 \mathrm{mAh} \mathrm{g}^{-1}$, the capacity retention is $70.8 \%$. To better illustrate the cyclic stability of the SGP-10-180-60 electrode, the electrode used PAA as a binder was prepared with the same ratio (where the GOPS was replaced with super P) and marked as Si-PAA-SP electrode. After 200 cycles, the specific capacity and capacity retention values were 584.6 mAh $\mathrm{g}^{-1}$ and 19.1\%, lower than the SGP-10-180-60 electrode (Fig. 1a). For comparison, the electrode with pure PEDOT:PSS as a binder (Si-PEDOT:PSS electrode) was also prepared. After 200 cycles, the specific capacity and capacity retention were $384.1 \mathrm{mAh} \mathrm{g}^{-1}$ and $13.6 \%$. What is more, it is clear that the Coulombic efficiency of SGP10-180-60 and Si-PEDOT:PSS electrodes is better than the Si-PAA-SP electrode (Fig. S3). This could be inferred by the dense structure of conductive polymer reducing the electrolyte permeation and the direct contact between electrolyte and $\mathrm{Si}$. The rate capabilities were tested in the range of 0.5-8.0 $\mathrm{A} \mathrm{g}^{-1}$ of SGP-10-180-60, Si-PEDOT:PSS, and Si-PAA-SP anodes (Fig. 1b). The SGP-10-180-60 showed the best rate capability, and the specific capacity could maintain $760 \mathrm{mAh} \mathrm{g}^{-1}$ at $8.0 \mathrm{~A} \mathrm{~g}^{-1}$, owing to the high electrical conductivity of PEDOT:PSS. The electrodes before and after cross-linking were tested with a quantitative lean electrolyte of $30 \mu \mathrm{L}$ and a fixed Li insertion capacity of $1000 \mathrm{mAh} \mathrm{g}^{-1}$ at $2.0 \mathrm{~A} \mathrm{~g} \mathrm{~g}^{-1}$ as shown in Fig. 1c. The electrode after crosslinking (SGP-10-180-60) can maintain this capacity for nearly 800 times compared with Si-PEDOT:PSS electrode that can maintain for less than 40 cycles and the Si-PAA-SP
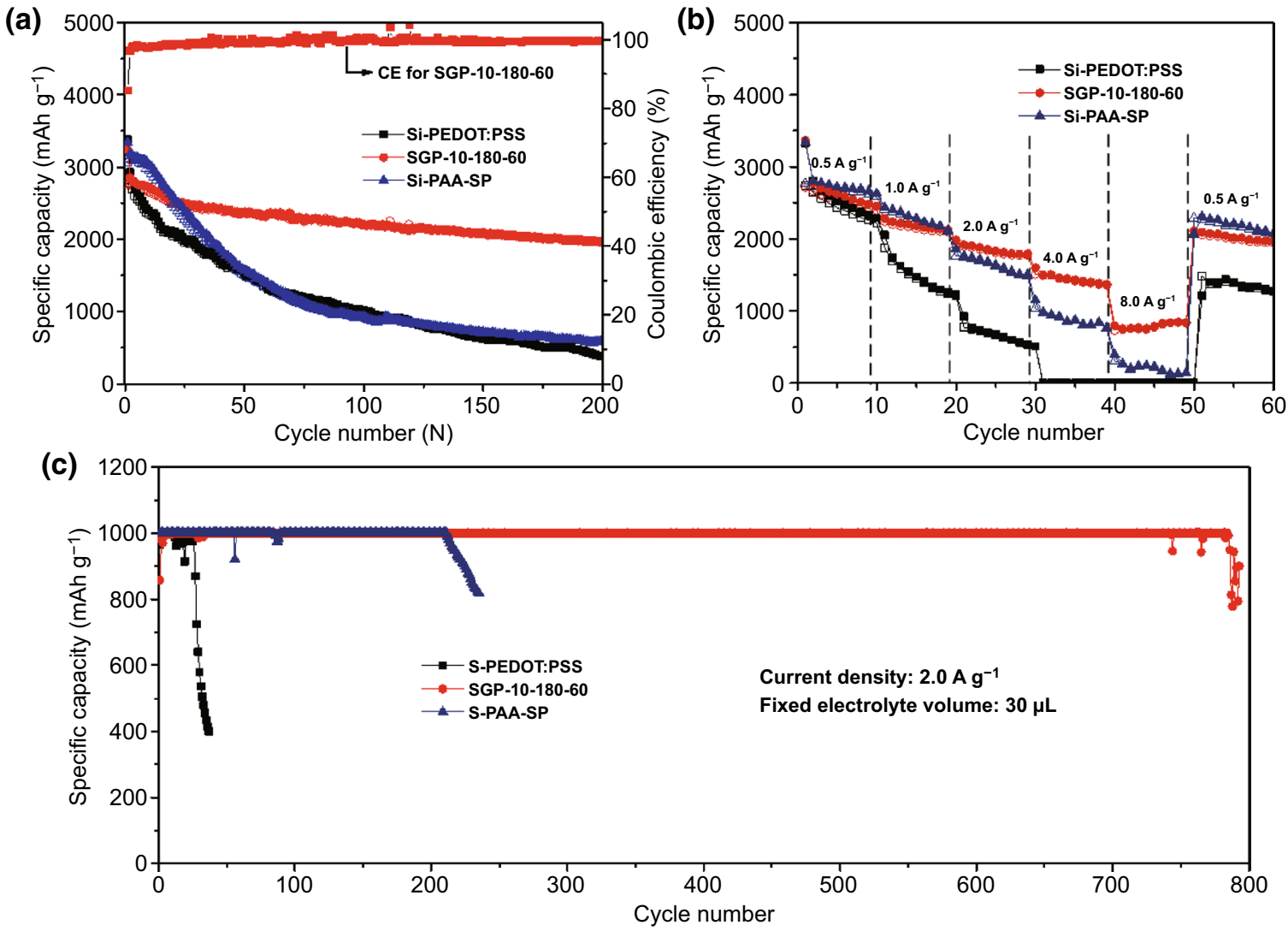

Fig. 1 a Galvanostatic cycling test of Si-PEDOT:PSS electrode, SGP-10-180-60 electrode and Si-PAA-SP anode at current density of 1.0 A $\mathrm{g}^{-1}$. b Rate capabilities of Si-PEDOT:PSS, SGP-10-180-60, and Si-PAA-SP anodes in current density of 0.5-8.0 A g ${ }^{-1}$. c Si-PEDOT:PSS, SGP-10180-60, and Si-PAA-SP electrodes tested with quantitative lean electrolyte of $30 \mu \mathrm{L}$ and a fixed Li insertion capacity of $1000 \mathrm{mAh} \mathrm{g}^{-1}$ at $2.0 \mathrm{~A}$ $\mathrm{g}^{-1}$ 
electrode for 220 times. The reason for such noteworthy capacity may be that the SGP-10-180-60 electrode can form a stable SEI after cross-linking.

\subsection{Mechanism of the Cross-linking Reaction}

To gain insight into the cross-linking reaction mechanism among PEDOT:PSS, GOPS, and Si nanoparticles, a series of XPS were conducted. As shown in Fig. 2a, broad peaks within the range of 102-105 eV are evident in both measured samples ascribed to the amorphous $\mathrm{SiO}_{\mathrm{x}}$ layer [29] . The conclusion agrees with the TEM results. In Fig. S1, the area ratio of oxidation state peaks has been increased after treatment with GOPS, indicating the interaction between $\mathrm{Si}$ and GOPS. Besides, the peaks centered at $\sim 99 \mathrm{eV}$ can be indexed to $\mathrm{Si}^{0}$, and there was a shift of $\sim 0.3 \mathrm{eV}$ toward high energy after treatment with GOPS. Figure $2 b$ shows the $S$ $2 p$ spectra consisting of two major parts, one from PEDOT at low binding energy (163-166 eV) and the other from PSS at high binding energy $(166-172 \mathrm{eV})[30,31]$. After treating with GOPS, the PSS region's $S 2 p$ spectra relate to the $-\mathrm{SO}_{3}{ }^{-}$groups interacting with $\mathrm{Na}^{+}$or $\mathrm{H}^{+}(\sim 169 \mathrm{eV})$ has a shift to higher binding energy, while the PSS interacting with PEDOT and PEDOT region stays constant. The XPS revealed the cross-linking between PEDOT:PSS and GOPS [32-34]. The O 1s spectra after treating with GOPS also showed a shift to high binding energy (Fig. 2c), suggesting the change in the chemical environment of the oxygen atoms after getting treated with GOPS.

FTIR measurement is conducted to confirm the cross-linking among $\mathrm{Si}$, GOPS, and PEDOT:PSS binder. As shown in Fig. 2d, after treated with GOPS, two distinct peaks located at $\sim 1200$ and $\sim 900 \mathrm{~cm}^{-1}$ were observed and assigned to $-\mathrm{CH}_{2}$ wagging, whereas the main band around $1100 \mathrm{~cm}^{-1}$ is due to Si-O-Si symmetric stretching mode [35], which is the strong evidence of cross-linking between Si and GOPS. From Fig. 2e, it can be seen that in the spectrum of the PEDOT:PSS after treatment with GOPS, there are two peaks centered at 1200 and $\sim 1080 \mathrm{~cm}^{-1}$ that also exist in GOPS and three peaks at 1130,1000 , and $920 \mathrm{~cm}^{-1}$ are consistent with pure PEDOT:PSS. As it can be seen that in pure GOPS spectra, there are two peaks at 820 and $910 \mathrm{~cm}^{-1}$, which can be assigned to epoxide moieties of GOPS [36]. After reaction with PEDOT:PSS, the two epoxide absorption bands disappeared, which further confirmed the reaction between GOPS and PEDOT:PSS. From the above analysis, GOPS acts as a bridge connecting Si with PEDOT:PSS. This structure is conducive to the contact between $\mathrm{Si}$ and binder and (a)

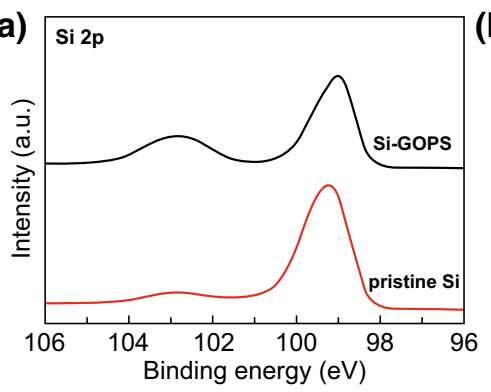

(d)

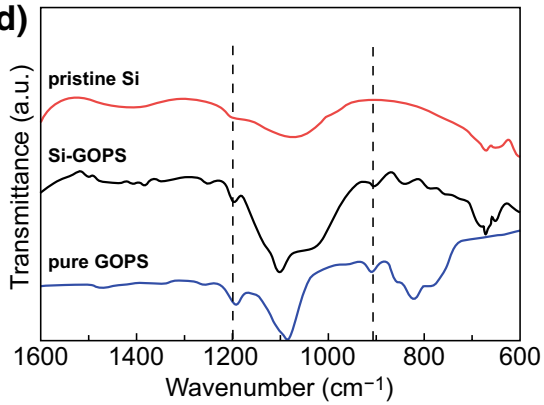

(b) $\mathrm{s} 2 \mathrm{p}$
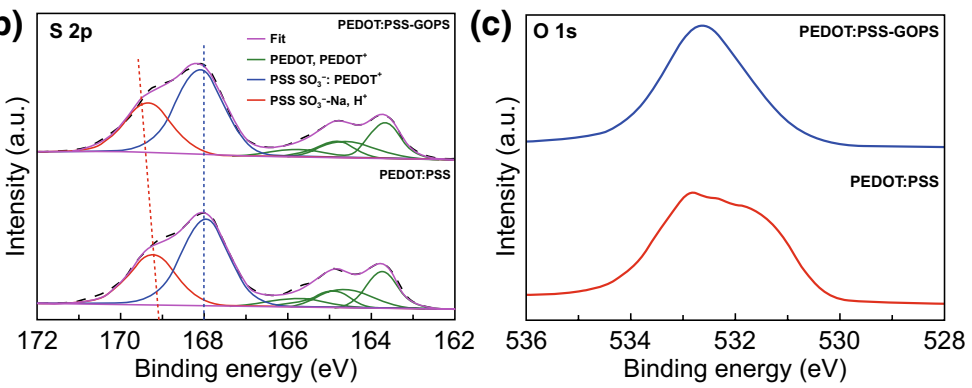

(e)

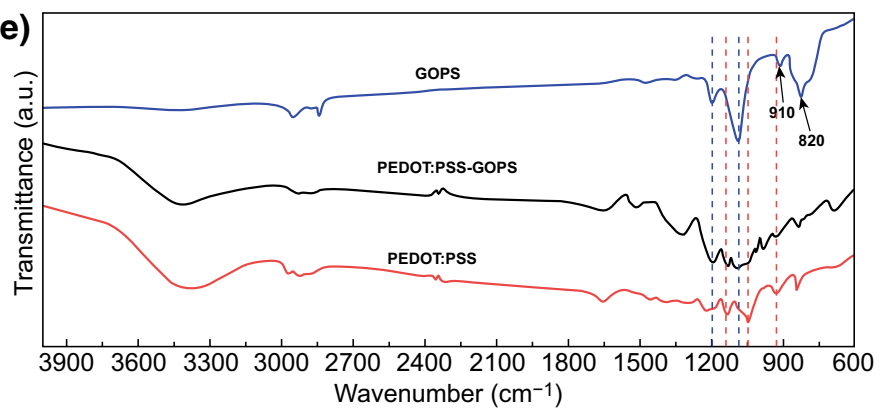

Fig. 2 XPS analysis: a Si 2p region of raw Si and after treatment by GOPS; b, c S 2p and O 1s spectrum of pure PEDOT:PSS and after treatment by GOPS. FTIR measurements of $\mathbf{d}$ raw Si NPs, pure GOPS, and Si after treatment with GOPS; e pure PEDOT:PSS, pure GOPS, and PEDOT:PSS after treatment by GOPS 
further facilitates a dense SEI film formation. Raman spectra of both pure PEDOT:PSS and after treatment by GOPS show the typical features of PEDOT:PSS [37]. There are no obvious Raman shifts of PEDOT:PSS after treatment with GOPS (Fig. S5), indicating that GOPS incorporation has not changed the main chain structure of PEDOT:PSS.

Based on the above characterizations, the cross-linking reactions between PEDOT:PSS, GOPS, and Si are shown in Fig. 3. The addition of ( $\gamma$-glycidyloxypropyl) trimethoxysilane (GOPS) in acidic PEDOT:PSS solution (1.4 wt\% in water, $\mathrm{pH}=2$ ) causes the epoxy groups to be opened to form two hydroxyl groups $(-\mathrm{OH})$ (chemical Eq. 1); these hydroxyl groups $(-\mathrm{OH})$ interact strongly with $-\mathrm{OSO}_{3} \mathrm{H}$ in PSS chains by hydrogen bonding as shown in chemical Eq. 2 [30]. The methoxyl groups $\left(-\mathrm{OCH}_{3}\right)$ on the other end of GOPS will be hydrolyzed, and the more stable silanol $(\mathrm{Si}-\mathrm{OH})$ groups are generated [38]. From Fig. S1, we can find that there is a layer of amorphous $\mathrm{SiO}_{\mathrm{x}}$ on $\mathrm{Si}$ nanoparticles, $\mathrm{Si}-\mathrm{OH}$ groups of hydrolyzed GOPS and surface of Si nanoparticles can react with each other releasing water molecules. In brief, GOPS finally realized the cross-linking of Si active material and binder PEDOT:PSS to form a spatial network structure, to increase the strength of the adhesive and the contact area between Si and PEDOT:PSS.

\subsection{Characterization of Different Electrodes After Cycling}

The impedance spectroscopy results of SGP-10-180-60, SiPEDOT:PSS, and Si-PAA-SP anodes after cycling 200 times at $1.0 \mathrm{~A} \mathrm{~g}^{-1}$ are shown in Fig. $4 \mathrm{a}$ (inset image is the corresponding fitting circuit model). The fitting resistances based on fitting equivalent are shown in Table S1. The EIS curves

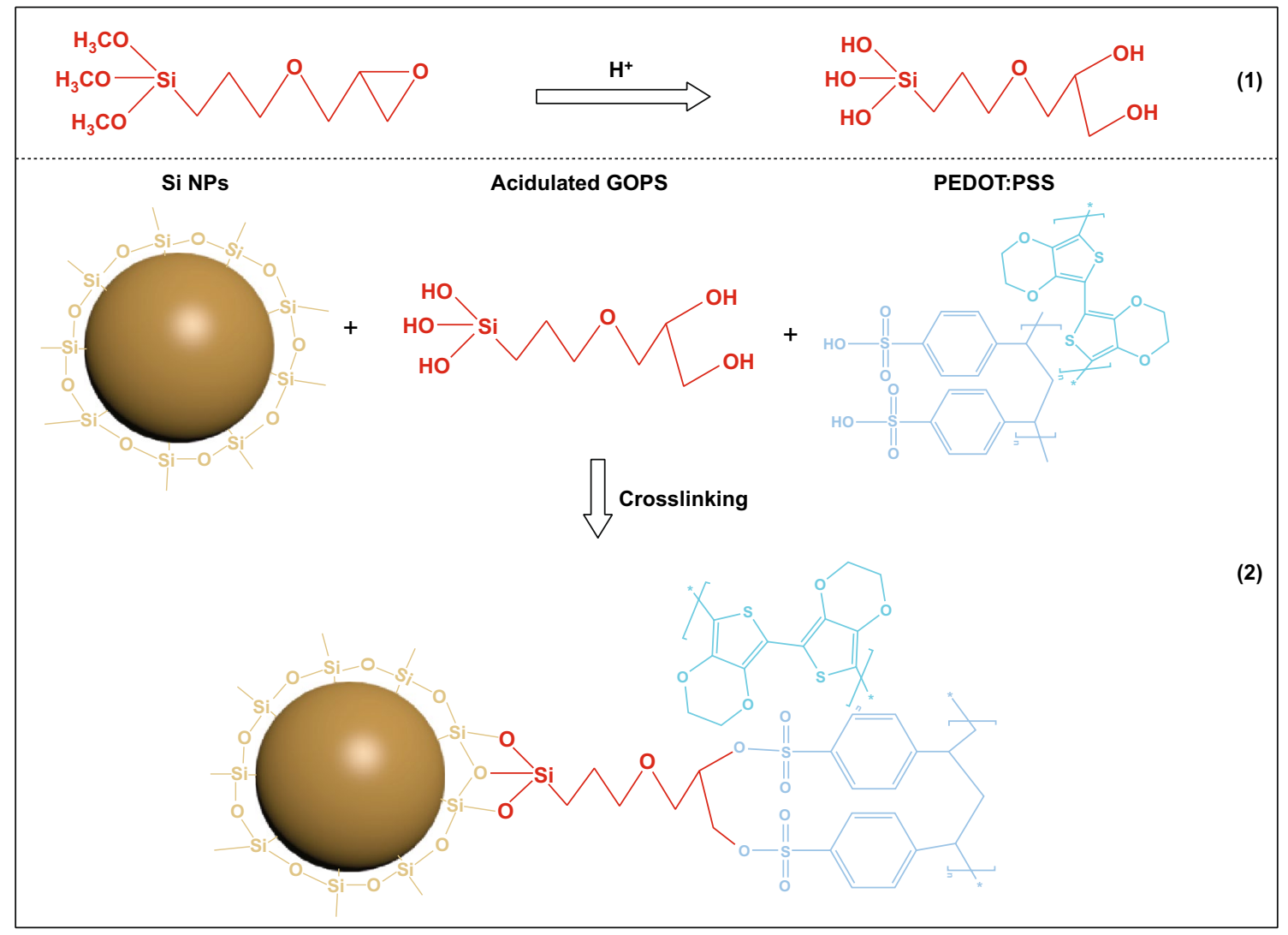

Fig. 3 Schematic diagram of the cross-linking interactions: (1) GOPS under acidic conditions; (2) cross-linking among Si nanoparticles, GOPS, and GOPS 
are composed of two semicircles (corresponding to $R_{\mathrm{SEI}}$ and $R_{\mathrm{ct}}$ resistances) in the medium-to-high frequency region and Warburg impedance $\left(\mathrm{Z}_{\mathrm{w}}\right)$. The SGP-10-180-60 electrode exhibited the smallest $R_{\mathrm{S}}$ and $R_{\mathrm{SEI}}$ resistances. To investigate electrodes' mechanical properties before and after crosslinking with GOPS, peeling tests were conducted as shown in Fig. 4b. According to the F-D curves, the peeling force of Si-PEDOT:PSS is $\sim 60 \mathrm{mN}$. After the addition of GOPS, the SGP-10-180-0 revealed a peeling force of $\sim 125 \mathrm{mN}$. After being sufficiently cross-linked with GOPS, the SGP10-180-60 presented the highest peeling force value of $~ 200$ $\mathrm{mN}$, indicating that GOPS cross-linking also encourages the improvement in the mechanical properties of the electrodes.

To better understand the cross-linked electrodes' improved cycling stability, the SEM was conducted for the Si-PAA-SP, Si-PEDOT:PSS, and SGP-10-180-60 electrodes before cycling and after 200 cycles at $1.0 \mathrm{~A} \mathrm{~g}^{-1}$ as shown in Fig. 5. It can be observed that Si nanoparticles are uniformly distributed on the Si-PAA-SP electrode and Si-PEDOT:PSS electrode (Fig. 5a, b). In comparison, there is a layer of polymer on the SGP-10-180-60 electrode (Fig. 5c). After 200 cycles at $1.0 \mathrm{~A} \mathrm{~g}^{-1}$, evident morphology changes are noticed on Si-PAA-SP and Si-PEDOT:PSS electrodes as marked by red circles in Fig. 5d, e, considered as pulverization of $\mathrm{Si}$ particles [39]. By contrast, no obvious detectable morphology change of Si nanoparticles was found for the SGP-10180-60 electrode after 200 cycles (Fig. 5f).

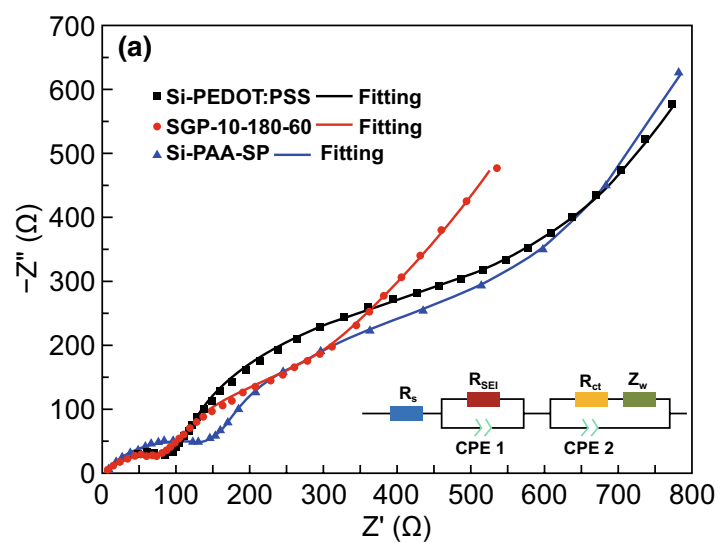

Figure 6 presents XPS $\mathrm{Ar}^{+}$etching depth analysis of Si-PEDOT:PSS and SGP-10-180-60 electrodes after 100 cycles with a quantitative lean electrolyte $(30 \mu \mathrm{L})$ and a fixed capacity of $1000 \mathrm{mAh} \mathrm{g}^{-1}$ at $2.0 \mathrm{~A} \mathrm{~g}^{-1}$ and the corresponding SEM images are shown in Fig. S6. The etching procedure included 6 steps, with an etching time of 500 s per step. Given that, the etch depth of $\mathrm{Ar}^{+}$is about $1.3 \mathrm{~nm}$ per $100 \mathrm{~s}$. Each etch step corresponded to $\sim 6.5 \mathrm{~nm}$. From Fig. 6a, b, the different behavior of two electrodes surfaces is obvious (etching time is $0 \mathrm{~s}$ ). The Si-PEDOT:PSS electrode showed no Si signal when the SGP-10-180-60 electrode displayed only the oxidation state of Si signal. It could be inferred that the surface of the electrodes after the cycling formed SEI. For the Si-PEDOT:PSS electrode, the SEI contained no Si element, while for the SGP-10-180-60 electrode, the Si of oxidized state $(102-104 \mathrm{eV})$ participated in the formation of the SEI. As can be deduced from the above analysis, the main state of raw $\mathrm{Si}$ NPs is $\mathrm{Si}^{0}(\sim 99 \mathrm{eV})$. Therefore, the $\mathrm{Si}$ of oxidized state on the surface of the electrode after cycling comes from GOPS. After etched one step (500 s), the Si signal was detected on the surface of the Si-PEDOT:PSS electrode, which was also confirmed by SEM images (Fig. S6a, b). After cycling, the Si-PEDOT:PSS electrode showed obvious cracks $(\sim 3.7 \mu \mathrm{m})$, revealing that the SEI had been destroyed and generated repeatedly, so the signal of active material Si was detected by etching only one step. As for the SGP-10-180-60 electrode, it can be seen from the SEM

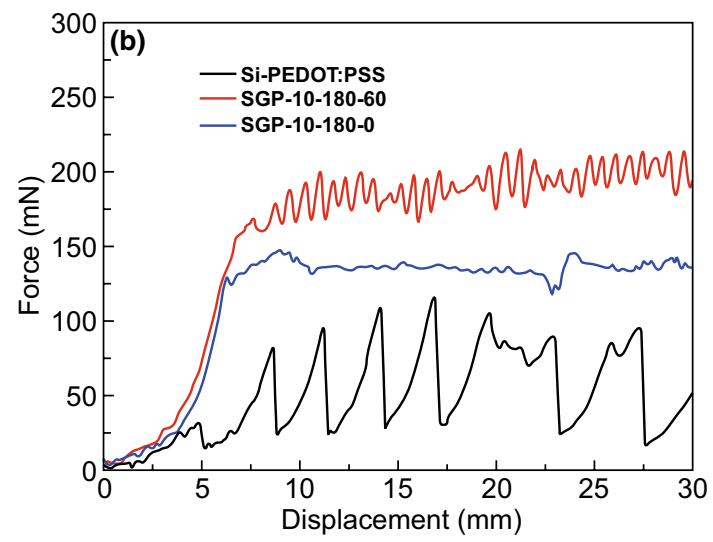

Fig. 4 a Nyquist plots of SGP-10-180-60, Si-PEDOT:PSS, and Si-PAA-SP anodes after 200 cycles at $1.0 \mathrm{~A} \mathrm{~g}^{-1}$. b Peeling tests of the different electrodes. F-D relation of SGP-10-180-0, SGP-10-180-60, and Si-PEDOT:PSS anodes 

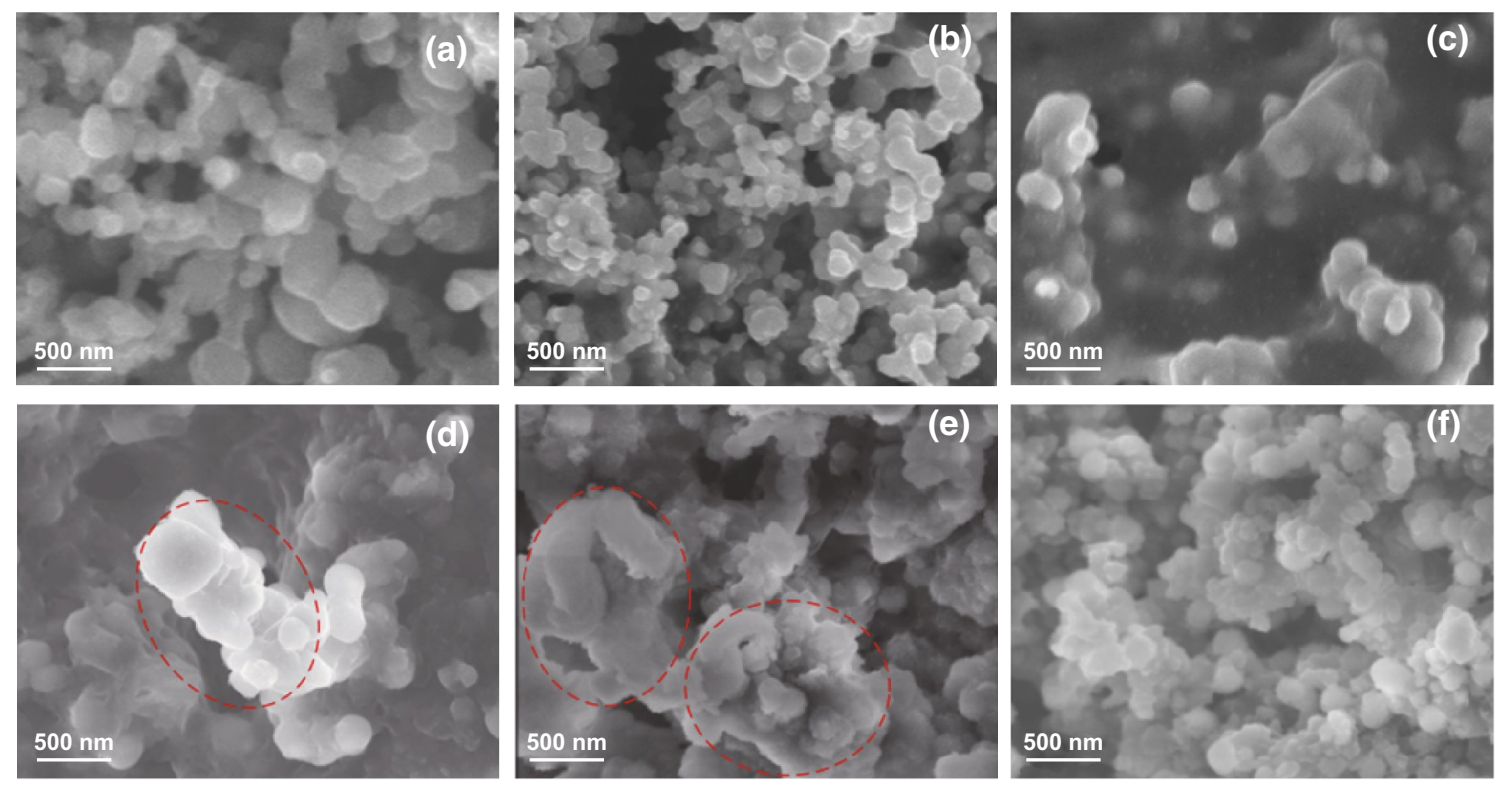

Fig. 5 SEM images of Si-PAA-SP (a, d), Si-PEDOT:PSS (b, e), and SGP-10-180-60 (c, f). Anodes before cycling (a-c) and after cycling 200 times at $1.0 \mathrm{~A} \mathrm{~g}^{-1}(\mathbf{d}-\mathbf{f})$

images (Fig. S6c, d) that the surface of the electrode after cycling is relatively uniform i.e., without obvious cracks. The signals of active material $\mathrm{Si}$ were detectable from the third steps of etching (1000 s). The XPS Ar ${ }^{+}$etching depth analysis peak fitting and relevant quantitative analysis of $\mathrm{Si}-\mathrm{Si}$ and $\mathrm{Si}-\mathrm{O}$ were added in Figs. S7, S8 and Table S2. With the increase in etching time, the $\mathrm{Si}-\mathrm{Si}$ bond content of Si-PEDOT:PSS electrode increased from 27 to $40 \%$, and after the etching $1500 \mathrm{~s}$, the content remained at $40 \%$. While the Si-Si content of the SGP-10-180-60 electrode gradually increased from 0 to $20 \%$ with the etching time increase from 0 to $3000 \mathrm{~s}$. It can be inferred that the addition of GOPS favors and contributes to forming a more stable SEI, which can alleviate the problems of SEI being destructed and repeated generation.

Overall, the mechanism is shown in Fig. 6c, the superior performance of SGP-10-180-60 electrodes can be attributed to the favorable properties of GOPS: (1) high conductivity and good film-forming properties of PEDOT:PSS are conducive to form a desired SEI on the Si surface; (2) GOPS as a typical cross-inker [30] can improve the stability, elasticity [40], mechanical stability [41], and adhesion [42] of
PEDOT:PSS; therefore, the incorporation of GOPS can better alleviate the volume expansion of $\mathrm{Si}$; (3) the interaction between the GOPS and Si improved the stability of Si electrode and made the Si not easy to fall off. (4) GOPS crosslinked PEDOT:PSS is conducive to the formation of a more stable SEI, which can suppress the continued consumption of electrolyte.

\subsection{Characterization of Full Batteries}

To further explore the practicability of the SGP-10-180-60 electrode, a full battery by a commercial Li-rich cathode was tested (NCM 523, SEM images of NCM 523 electrodes are shown in Fig. S9), as shown in Fig. 7a. The reversible capacity of NCM 523 cathode was $\sim 160 \mathrm{mAh} \mathrm{g}^{-1}$ at 2.8-4.25 V (Fig. S10); the mass loading employed was $10 \mathrm{mg} \mathrm{cm}^{-2}$, which is comparable to the cathode in practical mass loading of $15 \mathrm{mg} \mathrm{cm}^{-2}$ [43]. To balance this capacity, the SGP-10-180-60 anode had an active mass loading of only $0.8 \mathrm{mg} \mathrm{cm}^{-2}$. Figure $7 \mathrm{~b}$ overlays the potential curves of SGP-10-180-60 and NCM 523 half-cells. Figure 7c shows 
(a)

Si-PEDOT:PSS electrode (Si 2p)

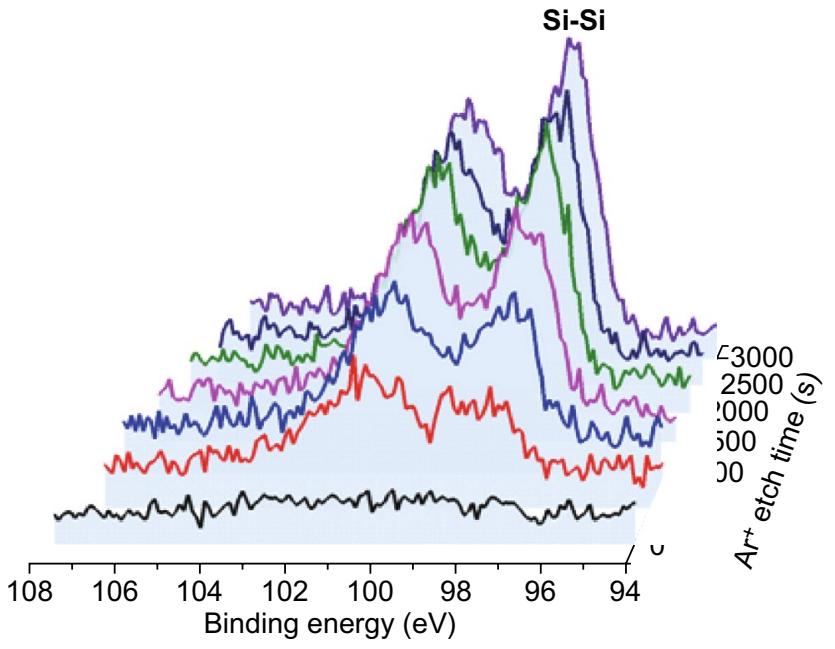

(b)

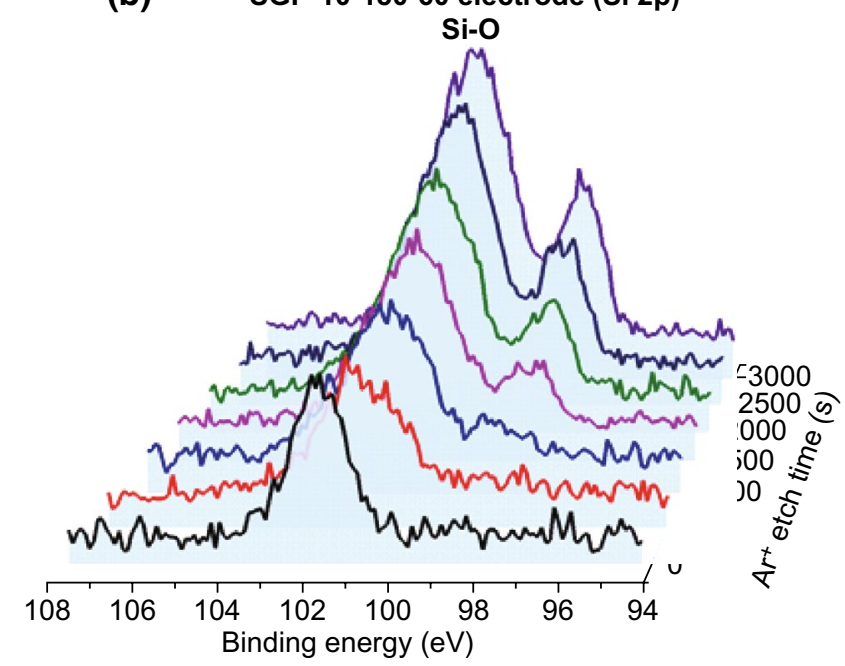

SGP-10-180-60 electrode (Si 2p)

(c)
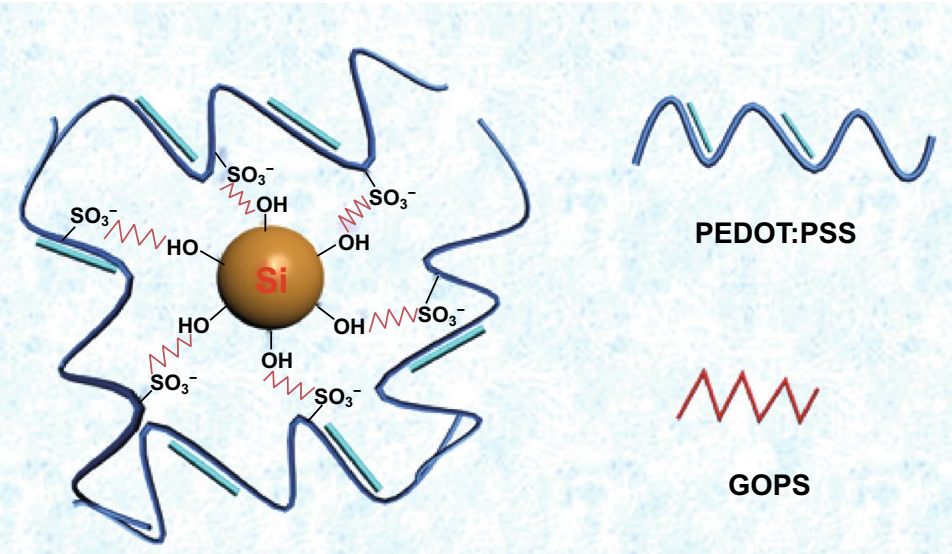

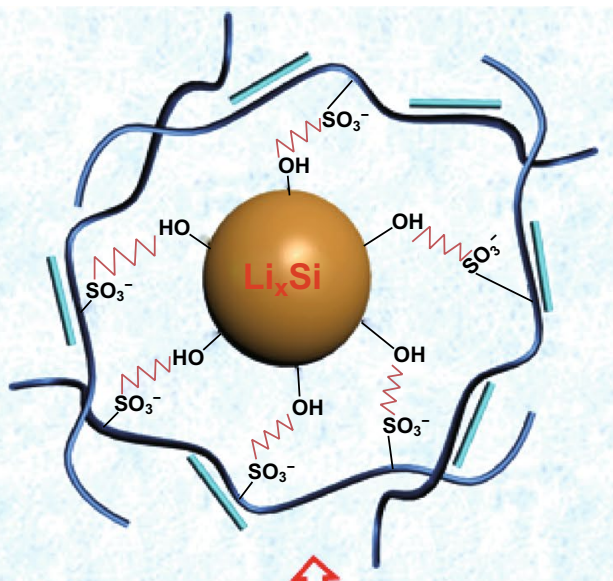

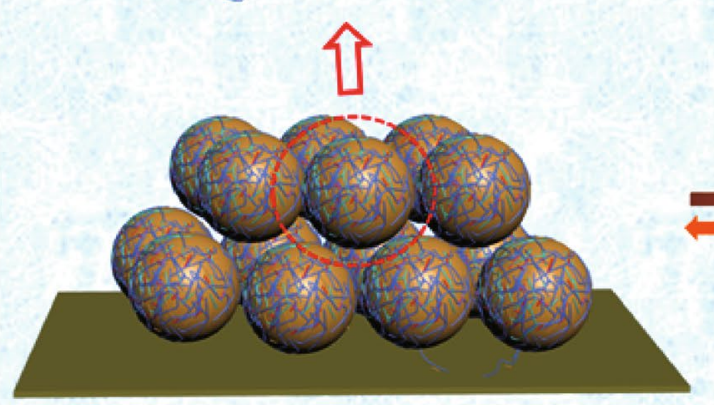

GOPS

\section{Lithiation}

Delithiation

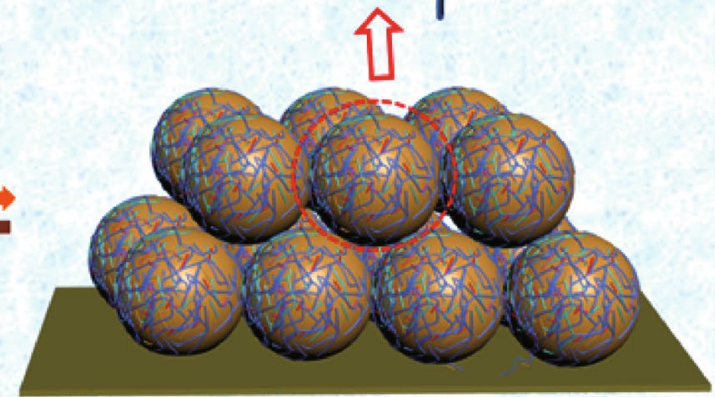

Fig. 6 Si $2 p$ spectra of XPS $\mathrm{Ar}^{+}$etching depth analysis of a Si-PEDOT:PSS and $\mathbf{b}$ SGP-10-180-60 electrodes after 100 cycles at 2.0 A g ${ }^{-1}$. Schematic illustration of the proposed mechanism explaining the electrochemical performance of SGP-10-180-60 anodes

the cycling performance of full battery at $20 \mathrm{~mA} \mathrm{~g}^{-1}$; the full battery delivers a high specific capacity of $\sim 1.5 \mathrm{mAh}$ $\mathrm{cm}^{-2}$ and exhibits a high ICE of $87.6 \%$ (after the initial three cycles, the Coulombic efficiency kept over 99\%). The full cell presented an average discharge voltage of $\sim 3.5 \mathrm{~V}$. The initial resultant specific energy was $530 \mathrm{Wh} \mathrm{kg}^{-1}$. After 60 cycles, it maintained $485 \mathrm{Wh} \mathrm{kg}^{-1}$, based on the total weight of anode and cathode. 

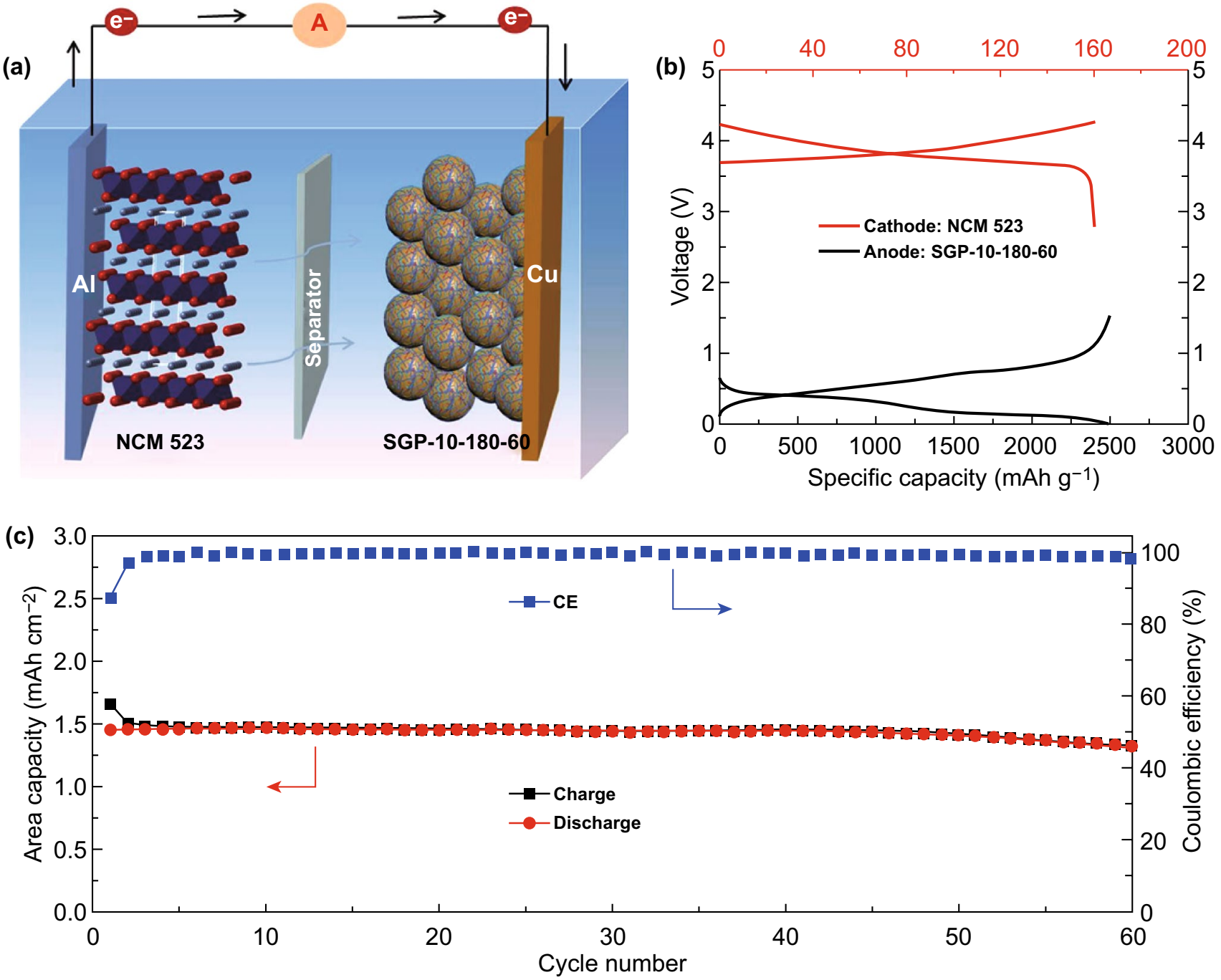

Fig. 7 a Diagram of full battery assembly, the mass loading $\left(10 \mathrm{mg} \mathrm{cm}^{-2}\right)$ of an NCM 523 cathode vs. a capacity balanced SGP-10-180-60 anode. b Typical cathode/anode cycling plots. c Cycling performance of full-cell at $20 \mathrm{~mA} \mathrm{~g}^{-1}$ with prelithiated SGP-10-180-60 anode and a NCM 523 cathode

\section{Conclusions}

In summary, we have designed a GOPS cross-linked PEDOT:PSS binder for Si electrodes. The cross-linked binder enhanced the contact between the $\mathrm{Si}$ and the binder and alleviated the volume expansion and contributed to the formation of a more stable and robust SEI to reduce the absorption of the electrolyte. The obtained SGP-10-180-60 electrode can maintain a high specific capacity of $1957.6 \mathrm{mAh} \mathrm{g}^{-1}$ after cycling 200 times, with a high capacity retention of $70.8 \%$. What is more, the
SGP-10-180-60 electrodes maintained up to $~ 800$ times when tested with a quantitative lean electrolyte of 30 $\mu \mathrm{L}$ and a fixed $\mathrm{Li}$ insertion capacity of $1000 \mathrm{mAh} \mathrm{g}^{-1}$ at $2.0 \mathrm{~A} \mathrm{~g}^{-1}$. SGP-10-180-60 anode $\|$ NCM 523 cathode full battery exhibited an area capacity of $\sim 1.5 \mathrm{mAh} \mathrm{cm}^{-2}$ and exhibited a high ICE of $87.6 \%$. The initial resultant specific energy was $530 \mathrm{Wh} \mathrm{kg}^{-1}$, and after 60 times, it maintained $485 \mathrm{Wh} \mathrm{kg}^{-1}$, based on the total weight of anode and cathode. This study paves a new way of designing binders used in other high-capacity electrodes with a strong volume effect. 
Acknowledgements The work is supported by Science and Technology Commission of Shanghai Municipality (20520710400), National Natural Science Foundation of China (21771124), Oceanic Interdisciplinary Program (project number SL2020MS020), and SJTU-Warwick Joint Seed Fund (2019/20) of Shanghai Jiao Tong University. Additionally, the authors thank the senior scientist Xue Ding from SJTU instrumental analysis center for her kind guidance regarding the XPS analysis.

Open Access This article is licensed under a Creative Commons Attribution 4.0 International License, which permits use, sharing, adaptation, distribution and reproduction in any medium or format, as long as you give appropriate credit to the original author(s) and the source, provide a link to the Creative Commons licence, and indicate if changes were made. The images or other third party material in this article are included in the article's Creative Commons licence, unless indicated otherwise in a credit line to the material. If material is not included in the article's Creative Commons licence and your intended use is not permitted by statutory regulation or exceeds the permitted use, you will need to obtain permission directly from the copyright holder. To view a copy of this licence, visit http://creativecommons.org/licenses/by/4.0/.

Electronic supplementary material The online version of this article (https://doi.org/10.1007/s40820-020-00564-5) contains supplementary material, which is available to authorized users.

\section{References}

1. G. Sandu, B. Ernould, J. Rolland, N. Cheminet, J. Brassinne et al., Mechanochemical synthesis of PEDOT:PSS hydrogels for aqueous formulation of Li-ion battery electrodes. ACS Appl. Mater. Interfaces 9(40), 34865-34874 (2017). https:// doi.org/10.1021/acsami.7b08937

2. B. Gendensuren, E.-S. Oh, Dual-crosslinked network binder of alginate with polyacrylamide for silicon/graphite anodes of lithium ion battery. J. Power Sources 384, 379-386 (2018). https://doi.org/10.1016/j.jpowsour.2018.03.009

3. B. Li, S. Li, Y. Jin, J. Zai, M. Chen et al., Porous Si@C ball-inball hollow spheres for lithium-ion capacitors with improved energy and power densities. J. Mater. Chem. A 6(42), 2109821103 (2018). https://doi.org/10.1039/c8ta07576j

4. B. Li, Z. Xiao, M. Chen, Z. Huang, X. Tie et al., Rice huskderived hybrid lithium-ion capacitors with ultra-high energy. J. Mater. Chem. A 5(46), 24502-24507 (2017). https://doi. org/10.1039/c7ta07088h

5. B. Li, R. Qi, J. Zai, F. Du, C. Xue et al., Silica wastes to highperformance lithium storage materials: a rational designed $\mathrm{Al}_{2} \mathrm{O}_{3}$ coating assisted magnesiothermic process. Small 12(38), 5281-5287 (2016). https://doi.org/10.1002/smll.20160 1914

6. B. Koo, H. Kim, Y. Cho, K.T. Lee, N.S. Choi et al., A highly cross-linked polymeric binder for high-performance silicon negative electrodes in lithium ion batteries. Angew. Chem. Int. Ed. 51(35), 8762-8767 (2012). https://doi.org/10.1002/ anie. 201201568

7. J.-H. Min, Y.-S. Bae, J.-Y. Kim, S.-S. Kim, S.-W. Song, Selforganized artificial sei for improving the cycling ability of silicon-based battery anode materials. Bull. Korean Chem. Soc. 34(4), 1296-1299 (2013). https://doi.org/10.5012/ bkcs.2013.34.4.1296

8. S. Jiang, B. Hu, R. Sahore, L. Zhang, H. Liu et al., Surfacefunctionalized silicon nanoparticles as anode material for lithium-ion battery. ACS Appl. Mater. Interfaces 10(51), 44924-44931 (2018). https://doi.org/10.1021/acsami.8b17729

9. D.S. Moock, S.O. Steinmuller, I.D. Wessely, A. Llevot, B. Bitterer et al., Surface functionalization of silicon, hopg, and graphite electrodes: toward an artificial solid electrolyte interface. ACS Appl. Mater. Interfaces 10(28), 24172-24180 (2018). https://doi.org/10.1021/acsami.8b04877

10. J. Li, N.J. Dudney, J. Nanda, C. Liang, Artificial solid electrolyte interphase to address the electrochemical degradation of silicon electrodes. ACS Appl. Mater. Interfaces 6(13), 10083-10088 (2014). https://doi.org/10.1021/am5009419

11. Z. Li, Y. Zhang, T. Liu, X. Gao, S. Li et al., Silicon anode with high initial coulombic efficiency by modulated trifunctional binder for high-areal-capacity lithium-ion batteries. Adv. Energy Mater. 10(20), 1903110 (2020). https://doi. org/10.1002/aenm.201903110

12. X. Liu, J. Zai, A. Iqbal, M. Chen, N. Ali et al., Glycerolcrosslinked PEDOT:PSS as bifunctional binder for si anodes: improved interfacial compatibility and conductivity. J. Colloid Interface Sci. 565(1), 270-277 (2020). https ://doi.org/10.1016/j.jcis.2020.01.028

13. H. Wu, G. Yu, L. Pan, N. Liu, M.T. McDowell et al., Stable Li-ion battery anodes by in-situ polymerization of conducting hydrogel to conformally coat silicon nanoparticles. Nat. Comm. 4, 1943 (2013). https://doi.org/10.1038/ncomm s2941

14. D. Liu, Y. Zhao, R. Tan, L.-L. Tian, Y. Liu et al., Novel conductive binder for high-performance silicon anodes in lithium ion batteries. Nano Energy 36, 206-212 (2017). https://doi. org/10.1016/j.nanoen.2017.04.043

15. J.S. Kim, W. Choi, K.Y. Cho, D. Byun, J. Lim et al., Effect of polyimide binder on electrochemical characteristics of surface-modified silicon anode for lithium ion batteries. J. Power Sources 244, 521-526 (2013). https://doi.org/10.1016/j.jpows our.2013.02.049

16. S.J. Park, H. Zhao, G. Ai, C. Wang, X. Song et al., Sidechain conducting and phase-separated polymeric binders for high-performance silicon anodes in lithium-ion batteries. J. Am. Chem. Soc. 137(7), 2565-2571 (2015). https://doi. org/10.1021/ja511181p

17. M. Döbbelin, R. Tena-Zaera, R. Marcilla, J. Iturri, S. Moya et al., Multiresponsive pedot-ionic liquid materials for the design of surfaces with switchable wettability. Adv. Funct. Mater. 19(20), 3326-3333 (2009). https://doi.org/10.1002/ adfm.200900863 
18. Y.H. Wijsboom, Y. Sheynin, A. Patra, N. Zamoshchik, R. Vardimon et al., Tuning of electronic properties and rigidity in PEDOT analogs. J. Mater. Chem. 21(5), 1368-1372 (2011). https://doi.org/10.1039/c0jm02679d

19. M. McGraw, P. Kolla, B. Yao, R. Cook, Q. Quiao, J. Wu, A. Smirnova, One-step solid-state in-situ thermal polymerization of silicon-PEDOT nanocomposites for the application in lithium-ion battery anodes. Polymer 99, 488-495 (2016). https ://doi.org/10.1016/j.polymer.2016.05.044

20. Y. Yao, N. Liu, M.T. McDowell, M. Pasta, Y. Cui, Improving the cycling stability of silicon nanowire anodes with conducting polymer coatings. Energy Environ. Sci. 5(7), 7927-7930 (2012). https://doi.org/10.1039/c2ee21437g

21. L. Yue, S. Wang, X. Zhao, L. Zhang, Nano-silicon composites using poly(3,4-ethylenedioxythiophene):Poly(styrenesulfon ate) as elastic polymer matrix and carbon source for lithiumion battery anode. J. Mater. Chem. 22(3), 1094-1099 (2012). https://doi.org/10.1039/c1jm14568a

22. T.M. Higgins, S.H. Park, P.J. King, C.J. Zhang, N. McEvoy et al., A commercial conducting polymer as both binder and conductive additive for silicon nanoparticle-based lithiumion battery negative electrodes. ACS Nano 10(3), 3702-3713 (2016). https://doi.org/10.1021/acsnano.6b00218

23. Y. Jin, B. Zhu, Z. Lu, N. Liu, J. Zhu, Challenges and recent progress in the development of $\mathrm{Si}$ anodes for lithium-ion battery. Adv. Energy Mater. 7(23), 1700715 (2017). https://doi. org/10.1002/aenm.201700715

24. J. Zhao, H.-W. Lee, J. Sun, K. Yan, Y. Liu et al., Metallurgically lithiated $\mathrm{SiO}_{\mathrm{x}}$ anode with high capacity and ambient air compatibility. Proc. Natl. Acad. Sci. USA. 113(27), 74087413 (2016). https://doi.org/10.1073/pnas.1603810113

25. X. Liu, A. Iqbal, N. Ali, R. Qi, X. Qian, Ion-cross-linkingpromoted high-performance Si/PEDOT:PSS electrodes: the importance of cations' ionic potential and softness parameters. ACS Appl. Mater. Interfaces 12(17), 19431-19438 (2020). https://doi.org/10.1021/acsami.0c00755

26. J. Sheng, K. Fan, D. Wang, C. Han, J. Fang et al., Improvement of the siox passivation layer for high-efficiency Si/PEDOT:PSS heterojunction solar cells. ACS Appl. Mater. Interfaces 6(18), 16027-16034 (2014). https://doi.org/10.1021/am503949g

27. W. Lu, C. Wang, W. Yue, L. Chen, Si/ PEDOT:PSS core/shell nanowire arrays for efficient hybrid solar cells. Nanoscale 3(9), 3631-3634 (2011). https://doi.org/10.1039/c1nr10629e

28. M. Pietsch, S. Jäckle, S. Christiansen, Interface investigation of planar hybrid n-Si/PEDOT:PSS solar cells with open circuit voltages up to $645 \mathrm{mV}$ and efficiencies of $12.6 \%$. Appl. Phys. A 115(4), 1109-1113 (2014). https://doi.org/10.1007/s0033 9-014-8405-4

29. G. Zheng, Y. Xiang, L. Xu, H. Luo, B. Wang et al., Controlling surface oxides in $\mathrm{Si} / \mathrm{C}$ nanocomposite anodes for high-performance li-ion batteries. Adv. Energy Mater. 8(29), 1801718 (2018). https://doi.org/10.1002/aenm.201801718

30. C.K. Chan, H. Peng, G. Liu, K. McIlwrath, X.F. Zhang et al., High-performance lithium battery anodes using silicon nanowires. Nat. Nano 3(1), 31-35 (2008). https://doi.org/10.1038/ nnano.2007.411
31. Y. Xia, K. Sun, J. Ouyang, Solution-processed metallic conducting polymer films as transparent electrode of optoelectronic devices. Adv. Mater. 24(18), 2436-2440 (2012). https ://doi.org/10.1002/adma.201104795

32. A. Håkansson, S. Han, S. Wang, J. Lu, S. Braun et al., Effect of (3-glycidyloxypropyl)trimethoxysilane (gops) on the electrical properties of PEDOT:PSS films. J. Polym. Sci. B-Polym. Phys. 55(10), 814-820 (2017). https://doi.org/10.1002/polb.24331

33. C. Liu, X. Du, S. Gao, A. Classen, A. Osvet et al., A crosslinked interconnecting layer enabling reliable and reproducible solution-processing of organic tandem solar cells. Adv. Energy Mater. 10(12), 1903800 (2020). https://doi.org/10.1002/ aenm.201903800

34. M. Solazzo, K. Krukiewicz, A. Zhussupbekova, K. Fleischer, M.J. Biggs et al., PEDOT:PSS interfaces stabilised using a pegylated crosslinker yield improved conductivity and biocompatibility. J. Mater. Chem. B 7(31), 4811-4820 (2019). https://doi.org/10.1039/c9tb01028a

35. P. Innocenzi, C. Figus, M. Takahashi, M. Piccinini, L. Malfatti, Structural evolution during evaporation of a 3-glycidoxypropyltrimethoxysilane film studied in situ by time resolved infrared spectroscopy. J. Phys. Chem. A 115(38), 10438-10444 (2011). https://doi.org/10.1021/jp204314b

36. M.U. Park, S.M. Lee, D.-W. Chung, Model system of crosslinked PEDOT: PSS adaptable to an application for an electrode with enhanced water stability. Synth. Metals 258, 116195 (2019). https://doi.org/10.1016/j.synthmet.2019.116195

37. T.P. Nguyen, P. Le Rendu, P.D. Long, S.A. De Vos, Chemical and thermal treatment of PEDOT:PSS thin films for use in organic light emitting diodes. Surf. Coat. Technol. 180-181, 646-649 (2004). https://doi.org/10.1016/j.surfc oat.2003.10.110

38. H. Ma, F. Cheng, J.Y. Chen, J.Z. Zhao, C.S. Li et al., Nest-like silicon nanospheres for high-capacity lithium storage. Adv. Matre. 19(22), 4067-4070 (2007). https://doi.org/10.1002/ adma.200700621

39. J. Song, M. Zhou, R. Yi, T. Xu, M.L. Gordin et al., Interpenetrated gel polymer binder for high-performance silicon anodes in lithium-ion batteries. Adv. Funct. Mater. 24(37), 5904-5910 (2014). https://doi.org/10.1002/adfm.201401269

40. Z.U. Khan, J. Edberg, M.M. Hamedi, G. Roger, G. Hjalmar et al., Thermoelectric polymers and their elastic aerogels. Adv. Mater. 28(22), 4556-4562 (2016). https://doi.org/10.1002/ adma.201505364

41. S. Zhang, P. Kumar, A.S. Nouas, L. Fontaine, H. Tang et al., Solvent-induced changes in PEDOT:PSS films for organic electrochemical transistors. APL Mater. 3(1), 014911 (2015). https://doi.org/10.1063/1.4905154

42. C. Duc, G.G. Malliaras, V. Senez, A. Vlandas, Long-term ageing of PEDOT:PSS: Wettability study. Synth. Met. 238, 14-21 (2018). https://doi.org/10.1016/j.synthmet.2018.02.003

43. W. Liu, H. Li, J. Jin, Y. Wang, Z. Zhang et al., Synergy of epoxy chemical tethers and defect-free graphene in enabling stable lithium cycling of silicon nanoparticles. Angew. Chem. Int. Ed. 131, 2-13 (2019). https://doi.org/10.1002/ange.201906612 